\title{
Colonization of polystyrene microparticles by Vibrio crassostreae: light and electron microscopic investigation
}

\author{
Foulon Valentin ${ }^{1}$, Le Roux Frederique ${ }^{2,3}$, Lambert Christophe ${ }^{1}$, Huvet Arnaud ${ }^{4}$, Soudant Philippe ${ }^{1}$, \\ Paul-Pont lka ${ }^{1, *}$
}

${ }^{1}$ Laboratoire des Sciences de l'Environnement Marin (LEMAR), UMR 6539 CNRS UBO IRD IFREMER

- Institut Universitaire Européen de la Mer, Technopôle Brest-Iroise - Rue Dumont d'Urville, 29280

Plouzané, France

2 Ifremer, Unité Physiologie Fonctionnelle des Organismes Marins, ZI de la Pointe du Diable, CS 10070,

F-29280 Plouzané, France

${ }^{3}$ Sorbonne Universités, UPMC Paris 06, CNRS, UMR 8227, Integrative Biology of Marine Models,

Station Biologique de Roscoff, CS 90074, F-29688, Roscoff cedex, France

${ }^{4}$ Ifremer, Laboratoire des Sciences de l'Environnement Marin (LEMAR, UMR 6539

UBO/CNRS/IRD/Ifremer), Centre Bretagne - ZI de la Pointe du Diable - CS 10070, 292804 Plouzané, France

* Corresponding author : Ika Paul-Pont, email address : ika.paulpont@univ-brest.fr

\begin{abstract}
:
Microplastics collected at sea harbour a high diversity of microorganisms including some Vibrio genus members, raising questions about the role of microplastics as a novel ecological niche for potentially pathogenic microorganisms. In the present study we investigated the adhesion dynamics of Vibrio crassostreae on polystyrene microparticles (micro-PS) using electronic and fluorescence microscopy techniques. Micro-PS were incubated with bacteria in different media (Zobell culture medium and artificial seawater) with or without natural marine aggregates. The highest percentage of colonised particles (38-100\%) was observed in Zobell culture medium, which may be related to nutrient availability for production of pili and exopolysaccharide adhesion structures. A longer bacterial attachment (6 days) was observed on irregular micro-PS compared to smooth particles $(<10 \mathrm{~h})$ but complete decolonisation of all particles eventually occurred. The presence of natural marine agreggates around micro-PS led to substantial and perennial colonisation featuring monospecific biofilms at the surface of the aggregates. These exploratory results suggest that $V$. crassostreae may be a secondary coloniser of micro-PS, requiring a multi-species community to form a durable adhesion phenotype. Temporal assessment of microbial colonisation on microplastics at sea using imaging and omics approaches are further indicated to better understand the microplastics colonisation dynamics and species assemblages.
\end{abstract}




\section{Graphical abstract}

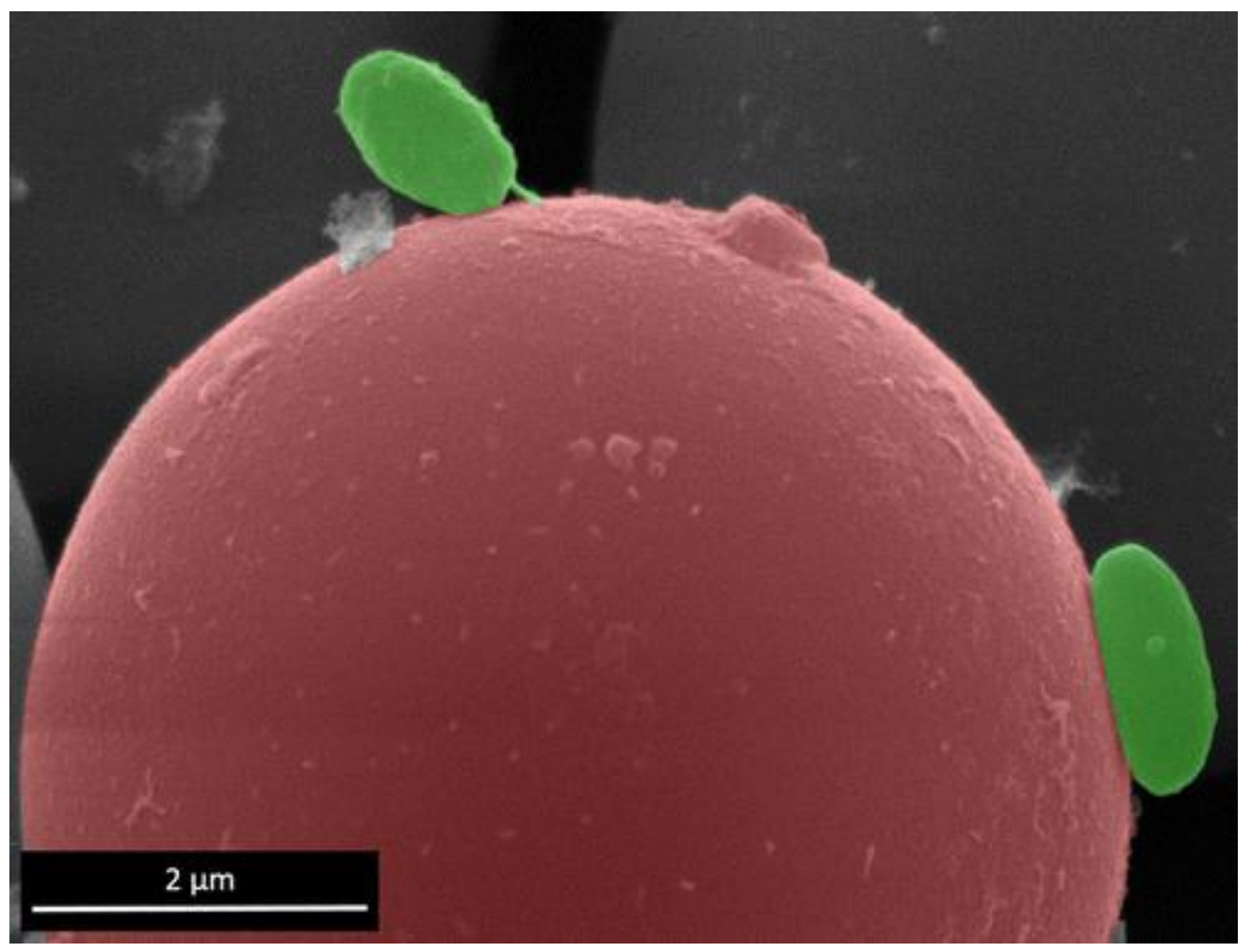

Keywords : microplastics, polystyrene, Vibrio, colonization 
43

44

45

46

47

48

49

50

51

52

53

54

55

56

57

58

59

60

61

62

63

64

65

66

67

\section{Introduction}

While global plastic production was less than 2 million tons in the 1950 s, worldwide production reached 311 million tons in $2014^{1}$. Plastic is a persistent material that accumulates in land and water ${ }^{2,3}$ and the latest estimates are up to 51 trillion floating plastic particles on the ocean surface ${ }^{4}$. Plastics can be divided into three classes: macroplastics $(>20 \mathrm{~cm})$, mesoplastics $(0.5-20 \mathrm{~cm})$ and microplastics $(<0.5 \mathrm{~cm})^{5}$. Primary microplastics are synthesized as microbeads which are used in many cosmetics, synthetic fibers used in clothing manufacture, and industrial preproduction pellets ${ }^{6}$. Fragmentation of macro- and mesoplastic waste by physico-chemical and biological processes results in secondary microplastics ${ }^{2,7}$.

Microplastics can be transported over time over long distances by ocean currents and thus are subjected to intense biofouling by prokaryotic and eukaryotic organisms ${ }^{8-13}$. As a consequence, microplastics are suspected to disperse potentially invasive and harmful species ${ }^{14}$, and may also represent a new ecological niche for microorganisms, also known as the "Plastisphere" ${ }^{10}$. Genomic studies on plastic samples collected in the Atlantic and Pacific oceans show a bacterial diversity that is different from the surrounding water ${ }^{13,15}$. Zettler et al. (2013) reported that a significant proportion of the bacteria on a polypropylene microplastic were vibrios (24\% of the total Operational Taxonomic Units of the plastic sample) ${ }^{10}$. The detection of Vibrionaceae members on marine microplastics was also demonstrated in other field studies conducted in the North Atlantic, the North Pacific Gyre, the Baltic Sea and the North Sea ${ }^{16-18}$.

Vibrios are ubiquitous marine bacteria that are ecologically and metabolically diverse members of both planktonic- and animal-associated microbial communities ${ }^{19,20}$ and they represent one of the best studied models for the ecology and evolution of bacterial populations in the wild ${ }^{21}$. The study of the distribution of vibrios on fine phylogenetic and spatial scales 
68

69

70

71

72

73

74

75

76

77

78

79

80

81

82

83

84

85

86

87

88

90

91

92

has demonstrated that vibrios coexisting in the water column can be divided into groups which pursue different lifestyles (free living, particle and animal-associated), defined as ecological populations ${ }^{22}$. Some populations are found in short lived blooms in the water column $^{21}$. Rapid growth of vibrios has been correlated with a diatom bloom ${ }^{23}$ or an association with algae ${ }^{24}$. This highlights, at microscale level, the importance of habitat occurrence and dynamic of vibrios population diversity in environment ${ }^{25,26}$. Vibrios encompass the well-studied human pathogen, $V$. cholerae, as well as some very important, albeit less thoroughly characterized, animal pathogens ${ }^{27}$. For example, vibrios may participate actively in repeated mortality outbreaks in oyster beds (Crassostrea gigas) in France with losses of up to $80-100 \%$ of production ${ }^{28,29}$. Vibrio species known for their pathogenic potential ( $V$. coralliilyticus, $V$. harveyi, $V$. splendidus, $V$. parahaemolyticus, $V$. alginolyticus and $V$. fluvialis) were detected on microplastics ${ }^{16,18}$, suggesting that microplastics may constitute a niche for vibrios, influencing their population dynamics and ultimately pathogen emergence.

Previous studies demonstrating the interactions between MP and vibrio have been based on one shot 16S metabarcoding targeting hypervariable regions of the small subunit of the ribosomal RNA (rRNA) gene ${ }^{10,16}$. However, this genomic approach at a single sampling time does not inform above the substrate specificity and the dynamics of the bacterial-plastic interaction. For instance, Datta et al. (2016) demonstrated that colonization of chitin microparticles result from a rapid succession of bacterial communities in which vibrios appear as secondary coloniser ${ }^{30}$. Vibrionaceae members are often stated to demonstrate habitat preference for plastics ${ }^{10,16,17}$ whereas the colonization dynamics are still unclear. Whether vibrios are first colonisers exhibiting specific affinity for plastic polymer substrates or secondary opportunistic colonisers dependant of other bacteria that present on plastics remains unknown. As little is known about the distribution and dispersal mechanisms of most 
93 pathogenic marine microbes, answering this question is of importance to understand the

94 environmental conditions precluding Vibrio attachment on marine particles and the relative

95 role of microplastics on their dispersal in regards with natural processes.

Vibrio crassostreae J2-9 has been associated to oyster disease (Pacific oyster Crassostrea gigas $^{28}$ ) but the environmental reservoir of this pathogen remain to be elucidate ${ }^{31}$. The aim of this study was to investigate the ability of this Vibrio strain to colonise polystyrene microplastics in different conditions using fluorescent and scanning electron microscopy over 4 days. The results suggested that Vibrio crassostreae J2-9 was a secondary coloniser whose association with microplastics was favoured by prior formation of natural multi-species marine aggregates around microplastic particles.

\section{Material and methods}

\section{Bacterial strain}

Vibrio crassostreae, strain J2-9, was isolated during an mortality event in C. gigas in the Bay of Brest, France in $2011^{28}$. A fluorescent-labelled bacterial strain constitutively expressing $g f p$ (Green Fluorescent Protein) from a stable plasmid was established ${ }^{32}$. To reduce experimental variation among assays, the same bacterial culture was divided into aliquots in $20 \%$ glycerol for cryopreservation at $-80^{\circ} \mathrm{C}$. Eighteen hours before each experiment, a preculture of J2-9 GFP was prepared in Zobell liquid medium (4g peptone, $1 \mathrm{~g}$ yeast extract, $0.1 \mathrm{~g}$ Iron Phosphate and 30g sea salt added per litter of milliQ water) ${ }^{33}$ supplemented with chloramphenicol $5 \mu \mathrm{g} \cdot \mathrm{mL}^{-1}$ at $18^{\circ} \mathrm{C}$ under mild stirring $(130 \mathrm{rpm})$. The pre-cultures were then analysed with a flow cytometer (FACSVerse, Becton Dickinson, San Jose, CA, USA) to assess bacterial growth and concentration. Bacteria were detected on the green fluorescence channel (FITC channel, 527 / 32nm) after excitation by the blue laser at $488 \mathrm{~nm}$, and the cell concentration was calculated based on the number of events (cells) recorded and the volume of samples analysed by the coupled Flow-sensor ${ }^{\mathrm{Tm}}$ (Supporting Information - Figure S1). 
119 Three types of polystyrene microparticles (micro-PS) were used as a colonization substrate:

120 (i) non-fluorescent smooth spherical microbeads (Phosphorex, Inc.) with a diameter of $6 \mu \mathrm{m}$

121 (PS-s); (ii) fluorescent smooth spherical microbeads (Phosphorex, Inc.; excitation/emission $122530 / 582 \mathrm{~nm}$ ) with a diameter of $5 \mu \mathrm{m}$ (PS-f); and (iii) non-fluorescent rough irregular particles 123 (Axalta) with a diameter of $45-60 \mu \mathrm{m}$ (PS-i).

125 Bacteria and micro-PS were incubated in $15 \mathrm{~mL}$ Pyrex glass culture tubes in order to

126 minimise plastic particles sticking to the flask walls. Similarly, samples were systematically

127 collected using glass Pasteur pipettes to avoid a significant decrease in micro-PS

128 concentration as demonstrated in preliminary tests using plastic pipettes (up to $80 \%$ reduction;

129 data not shown). Two experiments were performed to test: (i) the effects of micro-PS shape

130 (spherical and smooth vs. rough and irregular) in two different media (artificial seawater - sea

131 salt (Sigma-Aldrich, Missouri, US), and Zobell culture medium) on J2-9 GFP colonization

132 processes in axenic conditions (Experiment 1); and (ii) the presence of natural microbial

133 communities on J2-9 GFP colonization processes (multi-species condition) (Experiment 2).

134 3.1. Experiment 1 - Influence of micro-PS type in axenic conditions

135 Bacteria and micro-PS (PS-s, PS-f and PS-i) were incubated in either artificial seawater at $13635 \mathrm{~g} . \mathrm{L}^{-1}$ sea salt or in artificial seawater: Zobell media 50:50, all filtered at $0.22 \mu \mathrm{m}$ to remove 137 contaminating particles. Chloramphenicol was added to each medium at concentration of

$1385 \mu \mathrm{g} . \mathrm{L}^{-1}$ to maintain the selection pressure and discourage contaminants (e.g. other bacteria) to 139 interfere with the experiment. The initial J2-9 GFP bacterial concentration was adjusted to $1401.10^{6}$ bacteria per $\mathrm{mL}$ in a final volume of $7 \mathrm{~mL}$ of medium. The start of experiment 141 corresponds to the first contact between bacteria and microparticles, then all culture tubes 142 were immediately agitated at $300 \mathrm{rpm}$ at $22^{\circ} \mathrm{C}$. Experiment 1 was conducted in duplicate 
143 tubes, and was replicated 3 times. The percentage of particles colonized by one or more J2-9

144 GFP bacteria was evaluated by epifluorescence confocal microscopy every 15-30 minutes

145 during the first $10 \mathrm{~h}$ of contact, at $24 \mathrm{~h}$ post-incubation (PS-s and PS-f) and then every $24 \mathrm{~h}$ for

146 up to 4 days (PS-i only). For each sampling interval, 100 particles of PS-s and PS-f and 25

147 particles of PS-i were counted to estimate the percentage of colonized particles. Finally,

148 detailed observations on the interaction between J2-9 GFP and PS microbeads were recorded

149 using laser confocal microscopy and scanning electron microscopy (see details below $\S 4$ ).

\subsection{Experiment 2 - Colonization of micro-PS carrying a well-developed natural} marine aggregate by $\underline{\text { V. crassostreae }}$ J2-9 GFP

152 To better simulate environmental conditions encountered in situ, PS-s, PS-f and PS-i (100

153 particles per $\mathrm{mL}$ ) were incubated under gentle agitation for 7 days at $22^{\circ} \mathrm{C}$ in experimental

154 tanks containing freshly collected natural seawater from the Bay of Brest. Well-developed 155 aggregates were present around micro-PS within one week, as demonstrated in Wright et al.

$156(2013)^{34}$. Then, $7 \mathrm{~mL}$ of seawater containing micro-PS trapped in natural aggregates was

157 incubated with J2-9 GFP bacteria (initial concentration: $1.10^{6}$ bacteria per $\mathrm{mL}$ ) in glass tubes.

158 Tubes were placed at $22^{\circ} \mathrm{C}$ and gently stirred (300rpm) for up to 4 days. Qualitative

159 observation using epifluorescence confocal microscopy was performed every 30 minutes for 160 the first $10 \mathrm{~h}$ and then every $24 \mathrm{~h}$ until the end of the experiment (96h).

\subsection{Confocal microscopy}

163 Confocal microscopy allowed three dimensional visualization of micro-PS colonization by J2-

1649 GFP. This was monitored and measured using a Zeiss Axio Observer Z1 microscope (Carl

165 Zeiss SAS, Jena, Germany) equipped with a mercury vapor lamp, coupled to the confocal

166 module (spinning disc) VivaTome 3D. Observation of J2-9 GFP was performed by excitation

167 through a blue filter $494 / 20 \mathrm{~nm}$, and visualization of the green emission at 536/40 $\mathrm{nm}$ on the 
168 GFP channel. Fluorescent micro-PS (PS-f) were shown through a red emission at 628/40 nm

169 after excitation at 575/25 $\mathrm{nm}$. All other microparticles were visible in white light. Images of

170 particle colonization were obtained by a confocal laser scanning microscope (CLSM) Zeiss

171 LSM 780 using $488 \mathrm{~nm}$ and 561nm lasers. Colonized micro-PS were fixed with 6\%

172 glutaraldehyde for $24 \mathrm{~h}$ at $4^{\circ} \mathrm{C}$ after $1 \mathrm{~h} 30$ and $3 \mathrm{~h}$ interaction. CLSM observations were only

173 made in experiments performed in seawater, because there was green auto-fluorescence from

174 Zobell diluted medium.

175

\subsection{Scanning electron microscopy}

176 Suspensions of colonized PS particles were fixed in $6 \%$ glutaraldehyde in $0.1 \mathrm{M}$ sodium

177 cacodylate buffer $(1.75 \% \mathrm{w} / \mathrm{v}$ of $\mathrm{NaCl}, \mathrm{pH} 7.2)$. Suspensions were incubated for 10 minutes at

$1784^{\circ} \mathrm{C}$ before being filtered through polycarbonate filters with a $3 \mu \mathrm{m}$ pore size. Filters were

179 rinsed with a solution of sodium cacodylate $0.1 \mathrm{M}(2 \% \mathrm{w} / \mathrm{v}$ of $\mathrm{NaCl})$ in ultra-pure water

180 MilliQ. After rinsing, samples were dehydrated by successive immersions in alcoholic

181 hexamethyldisilazan (HMDS) (v:v): absolute ethanol:HMDS (3:1), absolute ethanol:HMDS

182 (1:1), absolute ethanol:HMDS (1:3), and pure HMDS. Finally, samples were coated with gold

183 palladium before being observed by scanning electron microscopy (SEM) (Hitachi S-3200N).

184 Images of the particle colonization were obtained after fixation after $1 \mathrm{~h} 30$ and $4 \mathrm{~h} 30$ of

185 interaction. Picture colorization was performed with the GNU Image Manipulation Program

186 (GIMP 2).

187 5. Statistical analysis

188 Non-parametric (Wilcoxon-Mann Whitney) and parametric tests (2 way Student's t test with 189 or without Welch correction) were carried out with R 3.2.3 data processing software (R Core 190 Team, 2015). Mean comparisons were carried out on the maximum of colonization and on the 
191 time needed to reach it between microparticles (PS-s, PS-f) and media (Zobell diluted media 192 and artificial seawater). A significant difference was observed for $\mathrm{p}<0.05$.

\section{Results and discussion}

194

\section{Fine scale micro-PS /Vibrio crassostreae interactions: pili formation and} exopolysaccharide production

Rapid movements of J2-9 GFP from one particle to another prior to bacterial adhesion were observed by confocal laser scanning microscopy (CLSM), suggesting active motility and rapid dispersal ability, which are essential for the attachment of bacteria to microparticles ${ }^{24}$, ${ }^{30,35}$. After $1 \mathrm{~h} 30$ of interaction, adherent J2-9 GFP cells were observed on $5 \mu \mathrm{m}$ fluorescent smooth spherical polystyrene microbeads (PS-f) (figure 1A). Scanning electron microscopy (SEM) observations confirmed the attachment of J2-9 bacteria to $6 \mu \mathrm{m}$ smooth spherical polystyrene microbeads (PS-s) via specific structures such as pili (arrow figure 1B). Pili are tiny filaments (5-7 $\mathrm{nm}$ in diameter for $1-2 \mu \mathrm{m}$ in length) linking bacteria to substrate by specific proteins (pilin polymers and adhesins) ${ }^{36-38}$. It is well known that pathogenic Vibrio species such as $V$. cholerae, $V$. parahaemolyticus, $V$. fulnificus and $V$. mimicus possess type IV pili that are essential for adherence, colonization and pathogenicity ${ }^{35,39,40}$. In these species, pili have predominantly a polar location, as it is observed here for $V$. crassostreae, even though lateral pili can be also observed. When cells appeared attached sideways on micro-PS, no specific structures were visible by SEM (figure 1C) suggesting that finer structures were probably implicated in adhesion. After $3 \mathrm{~h}$ of contact, CLSM observations showed a higher proportion of bead aggregates, with J2-9 GFP bacteria being located around PS-f particle aggregates (figure 1D). This was also confirmed by SEM observations made after $4 \mathrm{~h} 30$ of interaction, which demonstrated a high proportion of PS-s beads aggregated alongside J2-9, and the presence of fibrous exopolysaccharide-like structures (EPS) (figure 
215 1E). It was not possible to determine whether the presence of EPS was a cause or a

216 consequence of micro-PS aggregation. EPS such as glycocalyx secreted around the bacterial

217 wall are known to supplement reversible adhesion forces (electrostatic forces, Van der Waals,

218 hydrophobic or ionic interaction) and may play a crucial role in the first stage of adhesion ${ }^{41}$.

219 Datta et al. (2016) demonstrated the importance of particle attachment ability in the first hours

220 of contact (attachment step) at the expense of bacterial growth on substrate ${ }^{30}$. Even though

221 EPS may permit biofilm development leading to perennial settlement ${ }^{19,42,43}$, the smooth

222 micro-PS colonization observed in the present study was followed by a rather rapid

223 decolonization as described below (§ 2).

\section{4 \\ 2. Vibrio crassostreae exhibited rapid decolonization from smooth micro-PS}

225 Colonization dynamics for PS-s and PS-f in each medium are shown in Figure 2. In all

226 conditions (particle type and media) a dynamic in two phases was observed: first, the

227 percentage of colonized smooth micro-PS increased to a maximum between 29 minutes and 7

228 hours of contact; then a decolonization phase occurred with a decrease in the number of

229 colonized particles to zero after $24 \mathrm{~h}$ of contact.

230 In seawater, the mean percentage of colonized particles was below 14\% (except for one

231 analytical PS-f replicate). The maximum percentages of colonized PS-s were $4.0 \pm 1.4 \%, 14.5$

$232 \pm 3.5 \%$ and $6.0 \pm 1.4 \%$ (mean, $\mathrm{n}=2, \pm$ standard deviation, $\mathrm{SD}$ ) for experimental replicates 1,2

233 and 3, respectively (Figure 2A, Table S1). For PS-f, the maximum percentages of

234 colonization were between $1.0 \pm 1.4 \%$ and $1.5 \pm 0.7 \%$ (mean $\pm \mathrm{SD}$ ), except for replicate 3

235 that exhibited a higher colonization of $58.0 \pm 26.9 \%$ (Figure $2 \mathrm{~B}$, Table $\mathrm{S} 1$ ) despite identical

236 experimental conditions (temperature, agitation, media, culture age and state). We cannot

237 completely exclude experimental error to explain such high variability, but knowing that

238 all cautions have been taken to avoid them, these results suggest instead that other

239 parameters, such as fine level bacterial concentration dynamics, which were not controlled 
240 and not addressed here, may influence the regulation of bacterial adhesion. The maximum

241 percentage of colonized particles was significantly higher in Zobell culture medium than in

242 seawater both for PS-s (p-value=0.0003) and PS-f (p-value=0.0049) (Figure 3A, Table S1).

243 Colonization increased up to $38.0 \pm 2.8 \%, 72.0 \pm 11.3 \%$ and $95.5 \pm 3.5 \%$ for PS-s and

$24489.5 \pm 13.4 \%, 81 \pm 18.4$ and $78.5 \pm 9.2 \%$ for PS-f in Zobell culture medium (Figure 2A-B, Table

245 S1). No significant difference was observed between PS-s and PS-f for the maximum

246 percentage of colonized particles and the time needed to reach the maximum percentage of

247 colonization (Tm). Tm was between $4 \mathrm{~h} 20$ and $6 \mathrm{~h} 09$ in Zobell culture medium and this was

248 significantly higher than the Tm measured in seawater (28min to $2 \mathrm{~h} 45)$ for both PS-s ( $\mathrm{p}=$

$2490.0022)$ and PS-f $(p=0.0003)$ (Figure 3B, Table S2). Higher nutrient availability is known to

250 modulate the expression and maintenance of adhesion structures such as pili ${ }^{44}$ and thus could

251 enhance the final colonization success in Zobell culture medium with a higher percentage of

252 colonized particles. Environmental reservoir of Vibrio crassostreae J2-9 remain unknown ${ }^{31}$,

253 as well as its carbon source preference in natural environment. An earlier colonization was

254 observed in artificial seawater. The absence of nutrients may induce switch toward an active

255 substrate/nutrient research phenotype, meaning increase dispersal ability, adhesion tentative

256 or short adhesion on particulate matter present in the media (for instance microparticles).

257 Indeed, adhesion was reported as a survival strategy for vibrios in nutrient-limited natural

258 environment ${ }^{45}$, and better biofilm formation was reported in nutrient limiting condition than

259 in nutrient rich media ${ }^{46}$. Nutrient availability and/or quality might also explain the rapid

260 decolonization observed for all smooth micro-PS. Decolonization and dispersion is well

261 described in the literature for biofilms ${ }^{44,47}$ but is very poorly documented for the early stages

262 of adhesion, notably for vibrios. In artificial seawater, the only source of organic matter is

263 obtained from the bacterial inoculum by diluting the pre-culture, which was probably not

264 sufficient for the deposition of a primary/conditioning film on the bead surface as observed by 
265 CLSM and SEM for PS-s in Zobell culture medium (data not shown). The primary film 266 changes surface properties (hydrophobicity, polarity, surface tension) and could provide 267 sufficient nutrients at the substratum surface to be used by bacteria ${ }^{36}$. The absence of an 268 artificial seawater primary film could explain the lower overall colonization. In Zobell culture 269 medium, decolonization could be related to a limitation of some nutrients. Nutrient limitation 270 is reflected by stationary phase emergence in bacterial growth. However, no relationship was 271 demonstrated between the time of decolonization and the stationary phase of the culture. In 272 order to further address this question, nutrient controlled experimental systems like fermenters 273 equipped with a flow cell to measure bacterial concentration allowing maintenance of a stable 274 bacterial population and nutrient supply could be used. In addition, the measurement of the 275 numbers of bacteria per particle could not be quantitatively assessed in the present study by 276 using microscopy techniques, and the development of cytometry methods is encouraged to 277 assess this parameter as well as particle aggregation states, as demonstrated by Beloin et al. 278 (2008) and Geng et al. (2014) ${ }^{48,49}$.

279 Additionally to other factors, adhesion structures are known to be regulated by multifactorial 280 signals including quorum sensing ${ }^{41,43}$. Quorum sensing involves inter- and intraspecific 281 communication between bacterial cells by exchange of molecular signals involved in the 282 expression of target genes ${ }^{50,51}$. Therefore, an increase in cell density over time may regulate 283 quorum sensing signals and lead to the production of adhesion modulator compounds like 284 homoserine lactone causing cell detachment ${ }^{19,43,47}$. Physical parameters may also be 285 involved in the decolonization dynamics. Indeed, recent studies in bacterial adhesion forces 286 suggested that hydrodynamic movements associated with increased bacterial concentration 287 might lead to detachment from substrates ${ }^{52-54}$. This is in agreement with real time CLSM 288 observations that showed turbulences from rapid movement of bacteria. Collisions that 289 occurred between them may have then generated offsets of adherent bacteria. This hypothesis 
290 is also supported by the longer colonization observed for rough and irregular particles

291 (exposed in $\S 3$ ) whose interstices may have provided a shelter supporting bacterial

292 settlement.

\section{Long term colonization was observed on irregular micro-PS (PS-i)}

294

PS-i colonization started within the first hours of contact, and the maximum percentage of colonized particles was reached after $3 \mathrm{~h} 11$ of contact in Zobell diluted medium $(100 \%$ of particles colonized) and $2 \mathrm{~h} 54$ of contact in seawater ( $84 \% \pm 17$ of particles colonized) (Figure

2C) for one replicate. Particle colonization in the two other replicates was still increasing after

10h of contact in both media (Figure 2C). However, the percentage of colonized particles had decreased by the next sampling observation (24h), therefore the time for which the maximum percentage of colonized particles was reached was between $10 \mathrm{~h}$ and $24 \mathrm{~h}$ and cannot be assessed more precisely. PS-i colonization remained higher than $30 \%$ after $24 \mathrm{~h}$ of interaction in seawater for all replicates while a complete decolonization was observed in Zobell culture medium (data not shown). In seawater, even though a slight decrease of bacteria number per particle, PS-i particles remained colonized for up to six days, after which complete decolonization was observed. Substrate topography is a factor influencing bacterial adhesion 36, 55 . The presence of interstices on the irregular PS microparticles may have provided shelter for bacteria, as suggested by qualitative microscopical observations showing that bacteria were located in deep cavities on the PS-i. Bacteria were thus less exposed to hydrodynamic shearing forces ${ }^{55}$. The difference in size between PS-s $(6 \mu \mathrm{m})$ and PS-i $(45-60 \mu \mathrm{m})$ particles may also explain the difference in colonization intensity and duration. Even though the commercial polystyrene particles used in the present study are supposed to be free of additives or any other chemicals, their full composition is unknown and other chemicals could 313 also influence the patterns of colonization reported in this study. 
314 Overall, an efficient and lasting colonization with biofilm formation on micro-PS by $V$.

315 crassostreae J2-9 GFP was not observed, regardless of particle type and media. This may

316 suggest that this strain does not demonstrate any specific affinity for PS particles and is not

317 able to use particle resources, which is crucial for primary colonisers ${ }^{30}$. Consequently J2-9

318 may be more of a secondary coloniser, requiring other microbial communities (first

319 colonisers) that provide alternative carbon sources to durably colonise the polystyrene

320 surface. As the substrate specificity of Vibrio on synthetic polymers is still not investigated ${ }^{18}$,

321 further experiments using other vibrio strains and other plastic polymers (e.g. polyethylene

322 and polypropylene) should be conducted.

4. Natural aggregates enhanced perennial colonization of Vibrio crassostreae J2-9

324 PS-s, PS-f and PS-i incubated in natural seawater were rapidly subject to biofouling in the

325 first $24 \mathrm{~h}$ (figure 4A). After 7 days of incubation, no free micro-PS were detected in the natural

326 seawater. All micro-PS were entrapped in aggregates of significant size $( \pm 200 \mu \mathrm{m})$ formed by

327 debris and various microorganisms (figure 4B). Similar aggregates were also present with

328 identical characteristics (size, shape, appearance) in the control tank containing seawater only.

329 The rapid and efficient incorporation of the micro-PS within natural - probably multi-species -

330 aggregates observed here after 7 days of incubation in natural seawater is in accordance with

331 the rapid incorporation of micro-PS in monospecies marine phytoplankton aggregates (also

332 called marine snow) previously demonstrated under controlled laboratory conditions ${ }^{34,56,57}$.

333 Similarly, in the marine environment, plastic microparticles host a complex association of

334 prokaryotic and eukaryotic microbial communities ${ }^{8-10,12,13,16}$. Organism collaboration and

335 competition result in aggregate formation and trapping of plastic microparticles ${ }^{34,56,58}$.

336 When aggregates containing micro-PS were incubated with J2-9 GFP, a rapid and strong

337 colonization of the surfaces of the aggregates by J2-9 GFP was observed with some patches

338 of intense colonization (figure 4C). The vibrio J2-9 was located vertically in groups of 3 to 5 
339 cells forming corolla structures (figure 4D - head arrow), or was organized into monospecific

340 biofilms at the surface of the aggregates (figure 4C - head arrow). This possibly resulted from

341 (i) communication between J2-9 GFP and organisms in the aggregate and (ii) alternative

342 source of nutrients produced as by-products by the organisms in the aggregate. This

343 colonization on aggregates was durable and still observed after 96 hours of contact at higher

344 intensity compared to that observed in all particle types used in experiment 1 . However,

345 beyond time of contact (96h), it could not be established if J2-9 GFP disappearance was due

346 to loss of bacterial fluorescence or another factor. Predation of J2-9 GFP by ciliates feeding

347 on biofilms at the particle surface was observed as suggested by the appearance of

348 fluorescence in ciliates within a few hours (figure 4E). As J2-9 GFP was not visible

349 swimming in media, there was indirect evidence of lack of decolonization, contrary to all

350 other conditions (experiment 1 - particle colonization without pre-incubation in natural

351 seawater). Resulting of agitation, shear stress could also lead to an "erosion" of the aggregate

352 surface and the loss of J2-9 GFP bacteria. In natural environment, rafting communities on

353 plastic particles are exposed to currents and wave action, potentially resulting in their

354 dispersion. But shear stress was also reported as a factor which could increase cells' adhesion

355 capacity ${ }^{59}$, suggesting complex processes acting in colonization and decolonization.

356 The presence of natural aggregates around the particles seemed to have favoured the perennial

357 colonization of J2-9 GFP on micro-PS suggesting that $V$. crassostreae J2-9 might be

358 classified as a second coloniser of micro-PS, as it was demonstrated for Vibrionaceae on

359 chitin microparticles ${ }^{30}$. These authors suggested that most particle attached bacteria may be

360 secondary consumers that recycle waste products from primary consumers.

361 These exploratory results obtained under controlled laboratory conditions open new fields for

362 research addressing the dynamics of microplastic colonization by populations of vibrios. 
363 Indeed, our study concerning one strain of Vibrio and one polymer invite to investigate other

364 strains with other type of plastic. The presence of vibrios detected via genomic approaches on

365 microplastics collected at sea at one time point ${ }^{10,13,16,18}$ may result from secondary

366 colonization enabled by primary bacterial colonisers or more specific chemotactic attraction

367 to plastic polymer compounds. The identification of direct interaction as primary coloniser

368 using different synthetic polymers should be carefully assessed for both pathogenic, non-

369 pathogenic Vibrio species ${ }^{18}$. To test the hypothesis of secondary coloniser suggested by the

370 present in vitro study, further studies mimicking more closely the natural environment and

371 including omic approaches together with microscope observations, as it was done by Datta et

372 al. $(2016)^{30}$, are clearly required. Sequential sampling over time on different plastic polymers

373 and natural particles is necessary to characterize the temporal dynamics of first microbial -

374 eukaryote - and Vibrio - colonization in natural marine environments. If Vibrio are truly

375 second coloniser, the populations of microorganisms present on plastic and allowing for

376 Vibrio colonization must be characterized for various plastic polymers in different

377 environmental compartment and condition ${ }^{13-15}$. In addition, laboratory experiments using

378 diverse ecological populations of vibrios would be useful to investigate species relationships

379 (cooperation, competition, mutualism) involved in plastic colonization and their potential

380 effects upon ingestion by marine organisms ${ }^{22}$. Given the relatively low proportion of

381 microplastics in regards with other particulate matter (organic and inorganic) in marine

382 ecosystems ${ }^{60}$, the apparent complexity in the habitat preference of Vibrio species for plastic,

383 and the relative lack of knowledge regarding their natural dispersal mechanisms, its makes

384 difficult to postulate on the clear role of microplastics as vector for pathogenic vibrios in

385 marine environment ${ }^{13}$. Studies aiming to better understand the colonization dynamics of

386 microplastics by marine bacteria are required to investigate the role of microplastics as

387 vectors of harmful bacteria for marine organisms. 
The authors wish to thank Dr Philippe Elies for confocal laser scanning microscopy, Dr

390

Gérard Sinquin for scanning electron microscopy and Mr Claude Calvarin, glass blower, for revision of the English and his scientific comments on the manuscript.

\section{Supporting Information Available}

394

395

396

397

Figure S1 shows cytogram of fluorescent bacteria counting by flow cytometry. Tables S1 and $\mathrm{S} 2$ provide detailed statistical test results. This information is available free of charge via the Internet at http://pubs.acs.org.

\section{Figure captions}

Figure 1. Electronic and photonic microscopical observations of Vibrio crassostreae J2-9 GFP adhesion in PS microparticles. A, D: confocal laser scanning microscopy (CLSM) of the colonization of Vibrio crassostreae J2-9 GFP (green) on fluorescent polystyrene microparticles (PS-f) (red) in artificial seawater. B, C, E: scanning electron microscopy (SEM) observations of colonization of V. crassostreae J2-9 GFP (green) on polystyrene microparticles (PS-s) (red) in Zobell diluted media. SEM pictures were artificially colorized using the GIMP software. Sample fixation times were $1 \mathrm{~h} 30$ (A, B), 3h (D) and 4h30 (C, E). Scale bars: $2 \mu \mathrm{m}(\mathrm{A}, \mathrm{D}, \mathrm{E}) ; 1 \mu \mathrm{m}(\mathrm{B}) ; 0.5 \mu \mathrm{m}(\mathrm{C})$.

Figure 2. Percentage of colonized polystyrene microparticles by Vibrio crassostreae J2-9 GFP over time in seawater ( - blue) and Zobell diluted media $(\bigcirc$ - orange). These data were based on duplicate measurements from three independent experiments. For each sampling interval, 100 particles of PS-s and PS-f and 25 particles of PS-i were counted to estimate the 
410 percentage of colonized particles. A: smooth non fluorescent polystyrene microparticles (PS-

411 s); B: fluorescent polystyrene microparticles (PS-f); and C: irregular polystyrene

412 microparticles (PS-i).

413 Figure 3. A: maximum percentage of colonization (mean $\pm \mathrm{SD} ; \mathrm{n}=6$ ) for smooth polystyrene

414 microparticles (PS-s) and fluorescent polystyrene microparticles (PS-f) in both media

415 (artificial seawater and Zobell diluted media). B: time after which the maximum of

416 colonization was reached (mean $\pm \mathrm{SD} ; \mathrm{n}=6$ ) for PS-s and PS-f in both media. Letters $\mathrm{a}, \mathrm{b}$

417 indicate significant difference between groups, $\mathrm{p}<0.05$.

418 Figure 4. Smooth non fluorescent polystyrene microparticles (PS-s) colonization in natural

419 seawater. A: PS-s were rapidly subject to biofouling in the first $24 \mathrm{~h}$ observation - DAPI

420 staining. B: PS-s particles (shown by black arrows) entrapped in aggregates formed by debris

421 and microorganisms after 7 days of incubation. C: colonization of the aggregates containing

422 PS-s by Vibrio crassostreae J2-9 GFP after 8 hours of contact; PS-s are shown by black

423 arrows, J2-9 GFP biofilm is shown by arrowhead. D: corolla positioning of Vibrio

424 crassostreae J2-9 GFP on aggregates; PS-s are shown by black arrows, J2-9 GFP corolla

425 structure is shown by arrowhead. E: GFP fluorescent ciliate observed 2 hours after Vibrio

426 crassostreae J2-9 GFP addition. Scale bars: $10 \mu \mathrm{m}$ (A, E); $20 \mu \mathrm{m}$ (D); $50 \mu \mathrm{m}(\mathrm{B}, \mathrm{C})$.

\section{References}

428 1. PlasticsEurope, Plastics - the Facts 2015: An analysis of European plastics 429 production, demand and waste data. 2015.

$430 \quad$ 2. Barnes, D. K. A.; Galgani, F.; Thompson, R. C.; Barlaz, M., Accumulation and 431 fragmentation of plastic debris in global environments. Philosophical Transactions of the 432 Royal Society B-Biological Sciences 2009, 364 (1526), 1985-1998.

433 3. Thompson, R. C.; Moore, C. J.; vom Saal, F. S.; Swan, S. H., Plastics, the environment 434 and human health: current consensus and future trends. Philosophical Transactions of 435 the Royal Society B-Biological Sciences 2009, 364 (1526), 2153-2166.

$436 \quad 4$ van Sebille, E.; Wilcox, C.; Lebreton, L.; Maximenko, N.; Hardesty, B. D.; van 437 Franeker, J. A.; Eriksen, M.; Siegel, D.; Galgani, F.; Law, K. L., A global inventory of small 438 floating plastic debris. Environmental Research Letters 2015, 10 (12), 124006. 
439 5. Arthur, C.; Baker, J.; Bamford, H., (eds). 2009, Proceedings of the International 440 Research Workshop on the Occurrence, Effects, and Fate of Microplastic Marine Debris., 441 Sept 9-11, 2008, NOAA Technical Memorandum NOS-OR\&R-30.

442 6. Cole, M.; Lindeque, P.; Halsband, C.; Galloway, T. S., Microplastics as contaminants 443 in the marine environment: a review. Marine pollution bulletin 2011, 62 (12), 25884442597.

445 7. Sivan, A., New perspectives in plastic biodegradation. Current Opinion in 446 Biotechnology 2011, 22 (3), 422-426.

447 8. Harrison, J. P.; Sapp, M.; Schratzberger, M.; Osborn, A. M., Interactions Between 448 Microorganisms and Marine Microplastics: A Call for Research. Marine Technology 449 Society Journal 2011, 45 (2), 12-20.

$450 \quad$ 9. $\quad$ Carson, H. S.; Nerheim, M. S.; Carroll, K. A.; Eriksen, M., The plastic-associated 451 microorganisms of the North Pacific Gyre. Marine Pollution Bulletin 2013, 75 (1-2), 126452132.

453 10. Zettler, E. R.; Mincer, T. J.; Amaral-Zettler, L. A., Life in the "Plastisphere": 454 Microbial Communities on Plastic Marine Debris. Environmental Science \& Technology 455 2013, 47 (13), 7137-7146.

456 11. Harrison, J. P.; Schratzberger, M.; Sapp, M.; Osborn, A. M., Rapid bacterial 457 colonization of low-density polyethylene microplastics in coastal sediment microcosms. 458 Bmc Microbiologv 2014, 14, 232.

459 12. Reisser, J.; Shaw, J.; Hallegraeff, G.; Proietti, M.; Barnes, D. K. A.; Thums, M.; Wilcox, 460 C.; Hardesty, B. D.; Pattiaratchi, C., Millimeter-Sized Marine Plastics: A New Pelagic 461 Habitat for Microorganisms and Invertebrates. Plos One 2014, 9 (6), e100289. 462 13. Bryant, J. A.; Clemente, T. M.; Viviani, D. A.; Fong, A. A.; Thomas, K. A.; Kemp, P.; 463 Karl, D. M.; White, A. E.; DeLong, E. F., Diversity and Activity of Communities Inhabiting 464 Plastic Debris in the North Pacific Gyre. mSystems 2016, 1 (3), e00024-16.

465 14. Goldstein, M. C.; Carson, H. S.; Eriksen, M., Relationship of diversity and habitat 466 area in North Pacific plastic-associated rafting communities. Marine Biology 2014, 161 467 (6), 1441-1453.

468 15. Oberbeckmann, S.; Loeder, M. G. J.; Gerdts, G.; Osborn, A. M., Spatial and seasonal 469 variation in diversity and structure of microbial biofilms on marine plastics in Northern 470 European waters. Eems Microbiologv Ecologv 2014, 90 (2), 478-492.

471 16. Schmidt, V. T.; Reveillaud, J.; Zettler, E.; Mincer, T. J.; Murphy, L.; Amaral-Zettler, L. 472 A., Oligotyping reveals community level habitat selection within the genus Vibrio. 473 Frontiers in Microbiologv 2014, 5, 14.

474 17. De Tender, C. A.; Devriese, L. I.; Haegeman, A.; Maes, S.; Ruttink, T.; Dawyndt, P., 475 Bacterial community profiling of plastic litter in the Belgian part of the North Sea.

476 Environmental science \& technology 2015, 49 (16), 9629-9638.

477 18. Kirstein, I. V.; Kirmizi, S.; Wichels, A.; Garin-Fernandez, A.; Erler, R.; Löder, M.; 478 Gerdts, G., Dangerous hitchhikers? Evidence for potentially pathogenic Vibrio spp. on 479 microplastic particles. Marine Environmental Research 2016, 120, 1-8.

480 19. Thompson, F. L.; Iida, T.; Swings, J., Biodiversity of vibrios. Microbiology and 481 molecular biology reviews 2004, 68 (3), 403-431.

482 20. Thompson, J. R.; Polz, M. F., Dynamics of Vibrio Populations and Their Role in 483 Environmental Nutrient Cycling. 2006, ch13; p190-203. In Thompson F. Austin B. Swings I 484 (ed), The Biology of Vibrios. ASM Press, Washington, DC.

485 21. Takemura, A. F.; Chien, D. M.; Polz, M. F., Associations and dynamics of 486 Vibrionaceae in the environment, from the genus to the population level. Frontiers in 487 Microbiology 2014, 5, 38. 
488 22. Hunt, D. E.; David, L. A.; Gevers, D.; Preheim, S. P.; Alm, E. J.; Polz, M. F., Resource 489 partitioning and sympatric differentiation among closely related bacterioplankton.

490 Science 2008, 320 (5879), 1081-1085.

491 23. Gilbert, J. A.; Steele, J. A.; Caporaso, J. G.; Steinbrück, L.; Reeder, J.; Temperton, B.;

492 Huse, S.; McHardy, A. C.; Knight, R.; Joint, I., Defining seasonal marine microbial

493 community dynamics. The ISME journal 2012, 6 (2), 298-308.

494 24. Nealson, K. H.; Hastings, J. W., Quorum sensing on a global scale: massive

495 numbers of bioluminescent bacteria make milky seas. Applied and environmental

496 microbiology 2006, 72 (4), 2295-2297.

497 25. Szabo, G.; Preheim, S. P.; Kauffman, K. M.; David, L. A.; Shapiro, J.; Alm, E. J.; Polz,

498 M. F., Reproducibility of Vibrionaceae population structure in coastal bacterioplankton.

499 The ISME journal 2013, 7 (3), 509-519.

500 26. Vezzulli, L.; Grande, C.; Reid, P. C.; Hélaouët, P.; Edwards, M.; Höfle, M. G.; Brettar,

501 I.; Colwell, R. R.; Pruzzo, C., Climate influence on Vibrio and associated human diseases

502 during the past half-century in the coastal North Atlantic. Proceedings of the National

503 Academv of Sciences 2016, 113 (34), E5062-E5071.

504 27. Austin, B., Vibrios as causal agents of zoonoses. Veterinary Microbiology 2010,

505140 (3-4), 310-317.

506 28. Lemire, A.; Goudenège, D.; Versigny, T.; Petton, B.; Calteau, A.; Labreuche, Y.; Le

507 Roux, F., Populations, not clones, are the unit of vibrio pathogenesis in naturally infected

508 oysters. The ISME journal 2015, 9, 1523-1531.

509 29. Petton, B.; Bruto, M.; James, A.; Labreuche, Y.; Alunno-Bruscia, M.; Le Roux, F., 510 Crassostrea gigas mortality in France: the usual suspect, a herpes virus, may not be the 511 killer in this polymicrobial opportunistic disease. Frontiers in Microbiologv 2015, 6, 686.

512 30. Datta, M. S.; Sliwerska, E.; Gore, J.; Polz, M. F.; Cordero, O. X., Microbial interactions 513 lead to rapid micro-scale successions on model marine particles. Nature Communications $514 \quad 2016,7,11965$.

515 31. Le Roux, F.; Wegner, K. M.; Polz, M. F., Oysters and Vibrios as a Model for Disease 516 Dynamics in Wild Animals. Trends in microbiologv 2016, 24 (7), 568-580.

517 32. Le Roux, F.; Davis, B. M.; Waldor, M. K., Conserved small RNAs govern replication 518 and incompatibility of a diverse new plasmid family from marine bacteria. Nucleic Acids 519 Research 2011, 39 (3), 1004-1013.

520 33. Oppenheimer, C. H., The growth and viability of sixty-three species of marine 521 bacteria as influenced by hydrostatic pressure. Journal of Marine Research 1952, 11, 1052218.

523 34. Wright, S. L.; Thompson, R. C.; Galloway, T. S., The physical impacts of 524 microplastics on marine organisms: A review. Environmental Pollution 2013, 178, 483525492.

526 35. Nakasone, N.; Iwanaga, M., Pili of a Vibrio parahaemolyticus strain as a possible 527 colonization factor. Infection and Immunity 1990, 58 (1), 61-69.

528 36. Rubio, C., Compréhension des mécanismes d'adhésion des biofilms en milieu 529 marin en vue de la conception de nouveaux moyens de prévention. 2002, $p H D$ Thesis, 530 Université Paris 6. (http://archimer.ifremer.fr/doc/00000/222/).

531 37. Krebs, S. J.; Taylor, R. K., Protection and attachment of Vibrio cholerae mediated 532 by the toxin-coregulated pilus in the infant mouse model. Journal of bacteriology 2011, 533193 (19), 5260-5270.

$534 \quad 38 . \quad$ Lutz, C.; Erken, M.; Noorian, P.; Sun, S.; McDougald, D., Environmental reservoirs 535 and mechanisms of persistence of Vibrio cholerae. Frontiers in Microbiology 2013, 4, 536375. 
537 39. Frischkorn, K. R.; Stojanovski, A.; Paranjpye, R., Vibrio parahaemolyticus type IV 538 pili mediate interactions with diatom - derived chitin and point to an unexplored 539 mechanism of environmental persistence. Environmental microbiology 2013, 15 (5), $540 \quad 1416-1427$.

541 40. Tercero-Alburo, J. J.; González-Márquez, H.; Bonilla-González, E.; Quiñones-

542 Ramirez, E. I.; Vázquez-Salinas, C., Identification of capsule, biofilm, lateral flagellum, and 543 type IV pili in Vibrio mimicus strains. Microbial pathogenesis 2014, 76, 77-83.

544 41. Nadell, C. D.; Xavier, J. B.; Levin, S. A.; Foster, K. R., The evolution of quorum 545 sensing in bacterial biofilms. Plos Biologv 2008, 6 (1), 171-179.

546 42. Yildiz, F. H.; Visick, K. L., Vibrio biofilms: so much the same yet so different. Trends 547 in Microbiology 2009, 17 (3), 109-118.

548 43. Hammer, B. K.; Bassler, B. L., Quorum sensing controls biofilm formation in Vibrio 549 cholerae. Molecular Microbiologv 2003, 50 (1), 101-114.

$550 \quad$ 44. Sauer, K.; Cullen, M. C.; Rickard, A. H.; Zeef, L. A. H.; Davies, D. G.; Gilbert, P., 551 Characterization of nutrient-induced dispersion in Pseudomonas aeruginosa PA01 552 biofilm. Lournal of Bacteriologv 2004, 186 (21), 7312-7326.

553 45. Dawson, M. P.; Humphrey, B. A.; Marshall, K. C., Adhesion: a tactic in the survival 554 strategy of a marine vibrio during starvation. Current Microbiologv 1981, 6 (4), 195-199. 555 46. Petrova, O. E.; Sauer, K., Sticky situations: key components that control bacterial 556 surface attachment. Iournal of Bacteriologv 2012, 194 (10), 2413-2425.

557 47. Kaplan, J. B., Biofilm Dispersal: Mechanisms, Clinical Implications, and Potential

558 Therapeutic Uses. Lournal of Dental Research 2010, 89 (3), 205-218.

559 48. Beloin, C.; Houry, A.; Froment, M.; Ghigo, J. M.; Henry, N., A short-time scale 560 colloidal system reveals early bacterial adhesion dynamics. Plos Biology 2008, 6 (7), $561 \quad 1549-1558$.

562 49. Geng, J.; Beloin, C.; Ghigo, J.-M.; Henry, N., Bacteria Hold Their Breath upon

563 Surface Contact as Shown in a Strain of Escherichia coli, Using Dispersed Surfaces and

564 Flow Cytometry Analysis. Plos One 2014, 9 (7), e102049.

565 50. Cotter, P. A.; Stibitz, S., c-di-GMP-mediated regulation of virulence and biofilm

566 formation. Current Opinion in Microbiologv 2007, 10 (1), 17-23.

567 51. Rendueles, O.; Ghigo, J.-M., Mechanisms of Competition in Biofilm Communities.

568 Microbiology Spectrum 2015, 3 (3), MB-009-2014.

569 52. Gaboriaud, F.; Dufrene, Y. F., Atomic force microscopy of microbial cells:

570 Application to nanomechanical properties, surface forces and molecular recognition

571 forces. Colloids and Surfaces B-Biointerfaces 2007, 54 (1), 10-19.

572 53. Beaussart, A.; El-Kirat-Chatel, S.; Sullan, R. M. A.; Alsteens, D.; Herman, P.;

573 Derclaye, S.; Dufrene, Y. F., Quantifying the forces guiding microbial cell adhesion using

574 single-cell force spectroscopy. Nature Protocols 2014, 9 (5), 1049-1055.

575 54. Sullan, R. M. A.; Beaussart, A.; Tripathi, P.; Derclaye, S.; El-Kirat-Chatel, S.; Li, J. K.;

576 Schneider, Y. J.; Vanderleyden, J.; Lebeer, S.; Dufrene, Y. F., Single-cell force spectroscopy

577 of pili-mediated adhesion. Nanoscale 2014, 6 (2), 1134-1143.

578 55. Taylor, R. L.; Verran, J.; Lees, G. C.; Ward, A. J. P., The influence of substratum

$\underline{579}$ topography on bacterial adhesion to polymethyl methacrylate. Journal of Materials $580 \quad$ Science-Materials in Medicine 1998, 9 (1), 17-22.

$581 \quad 56 . \quad$ Long, M.; Moriceau, B.; Gallinari, M.; Lambert, C.; Huvet, A.; Raffray, J.; Soudant, P., 582 Interactions between microplastics and phytoplankton aggregates: Impact on their 583 respective fates. Marine Chemistrv 2015, 175, 39-46.

$584 \quad 57 . \quad$ Lagarde, F.; Olivier, O.; Zanella, M.; Daniel, P.; Hiard, S.; Caruso, A., Microplastic

585 interactions with freshwater microalgae: Hetero-aggregation and changes in plastic 
586 density appear strongly dependent on polymer type. Environmental Pollution 2016, 215, 587 331-339.

588 58. Grossart, H.-P.; Kiørboe, T.; Tang, K.; Ploug, H., Bacterial colonization of particles: 589 growth and interactions. Anolied and Environmental Microbiologv 2003, 69 (6), 35005903509.

591 59. Persat, A.; Nadell, C. D.; Kim, M. K.; Ingremeau, F.; Siryaporn, A.; Drescher, K.;

592 Wingreen, N. S.; Bassler, B. L.; Gitai, Z.; Stone, H. A., The mechanical world of bacteria. Cell 593 2015, $161(5), 988-997$.

594 60. Koelmans, A. A.; Bakir, A.; Burton, G. A.; Janssen, C. R., Microplastic as a vector for 595 chemicals in the aquatic environment: critical review and model-supported

596 reinterpretation of empirical studies. Environmental science \& technology 2016, 50 (7), 597 3315-3326.

598 


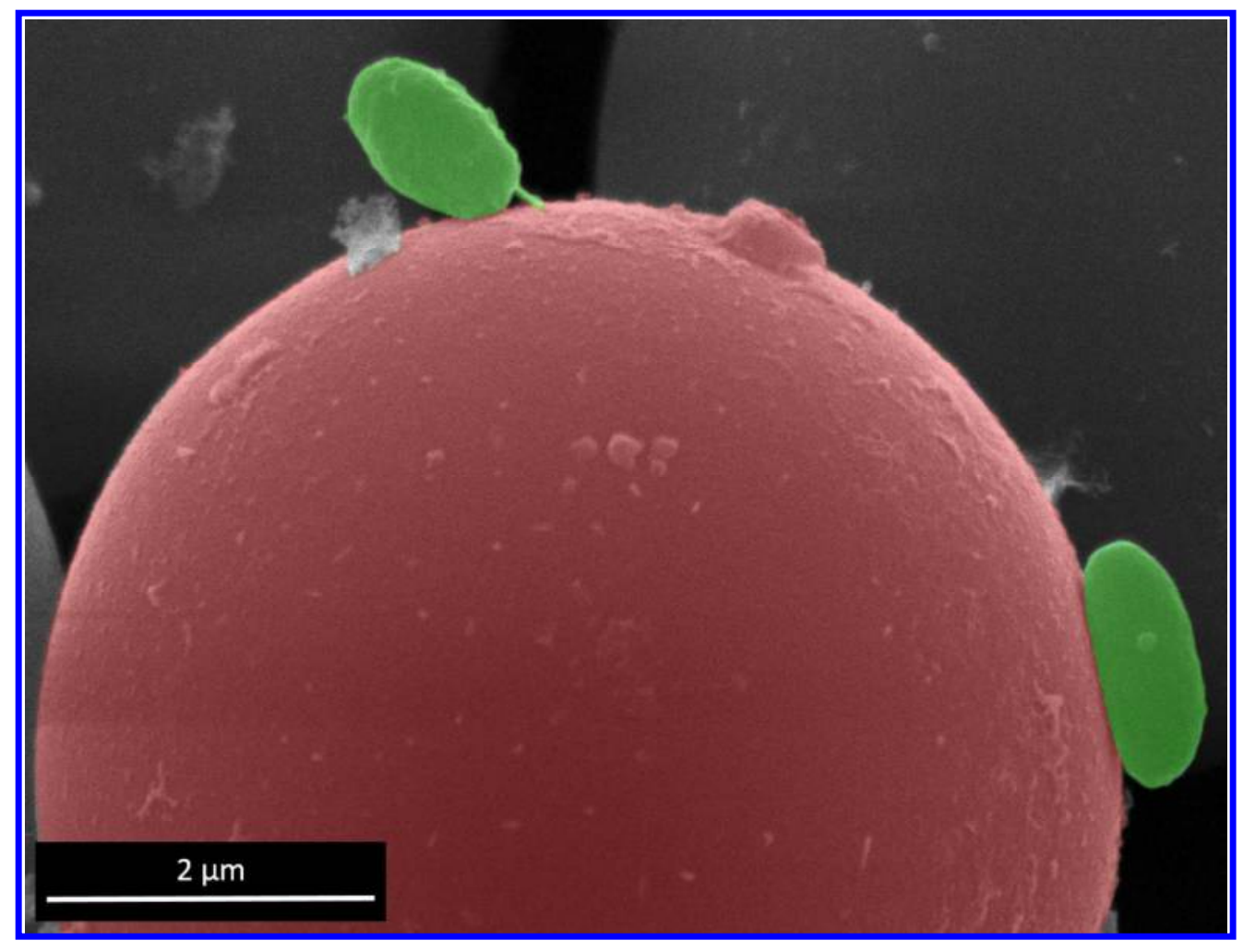

TOC abstract graphic

$229 \times 174 \mathrm{~mm}(150 \times 150 \mathrm{DPI})$ 


\section{Figure captions}

Figure 1. Electronic and photonic microscopical observations of Vibrio crassostreae J2-9 GFP adhesion in PS microparticles. A, D: confocal laser scanning microscopy (CLSM) of the colonization of Vibrio crassostreae J2-9 GFP (green) on fluorescent polystyrene microparticles (PS-f) (red) in artificial seawater. B, C, E: scanning electron microscopy (SEM) observations of colonization of V. crassostreae J2-9 GFP (green) on polystyrene microparticles (PS-s) (red) in Zobell diluted media. SEM pictures were artificially colorized using the GIMP software. Sample fixation times were $1 \mathrm{~h} 30$ (A, B), 3h (D) and $4 \mathrm{~h} 30$ (C, E). Scale bars: $2 \mu \mathrm{m}(\mathrm{A}, \mathrm{D}, \mathrm{E}) ; 1 \mu \mathrm{m}(\mathrm{B}) ; 0.5 \mu \mathrm{m}(\mathrm{C})$.

Figure 2. Percentage of colonized polystyrene microparticles by Vibrio crassostreae J2-9 GFP over time in seawater $(-$ blue $)$ and Zobell diluted media $(\bigcirc$ - orange). These data were based on duplicate measurements from three independent experiments. For each sampling interval, 100 particles of PS-s and PS-f and 25 particles of PS-i were counted to estimate the percentage of colonized particles. A: smooth non-fluorescent polystyrene microparticles (PSs); B: fluorescent polystyrene microparticles (PS-f); and C: irregular polystyrene microparticles (PS-i).

Figure 3. A: maximum percentage of colonization (mean $\pm \mathrm{SD} ; \mathrm{n}=6$ ) for smooth polystyrene microparticles (PS-s) and fluorescent polystyrene microparticles (PS-f) in both media (artificial seawater and Zobell diluted media). B: time after which the maximum of colonization was reached (mean $\pm \mathrm{SD} ; \mathrm{n}=6$ ) for PS-s and PS-f in both media. Letters $\mathrm{a}, \mathrm{b}$ indicate significant difference between groups, $\mathrm{p}<0.05$.

Figure 4. Smooth non-fluorescent polystyrene microparticles (PS-s) colonization in natural seawater. A: PS-s were rapidly subject to biofouling in the first $24 \mathrm{~h}$ observation - DAPI staining. B: PS-s particles (shown by black arrows) entrapped in aggregates formed by debris and microorganisms after 7 days of incubation. C: colonization of the aggregates containing PS-s by Vibrio crassostreae J2-9 GFP after 8 hours of contact; PS-s are shown by black arrows, J2-9 GFP biofilm is shown by arrowhead. D: corolla positioning of Vibrio crassostreae J2-9 GFP on aggregates; PS-s are shown by black arrows, J2-9 GFP corolla structure is shown by arrowhead. E: GFP fluorescent ciliate observed 2 hours after Vibrio crassostreae J2-9 GFP addition. Scale bars: $10 \mu \mathrm{m}$ (A, E); $20 \mu \mathrm{m}$ (D); $50 \mu \mathrm{m}$ (B, C). 


\section{Figure 1}
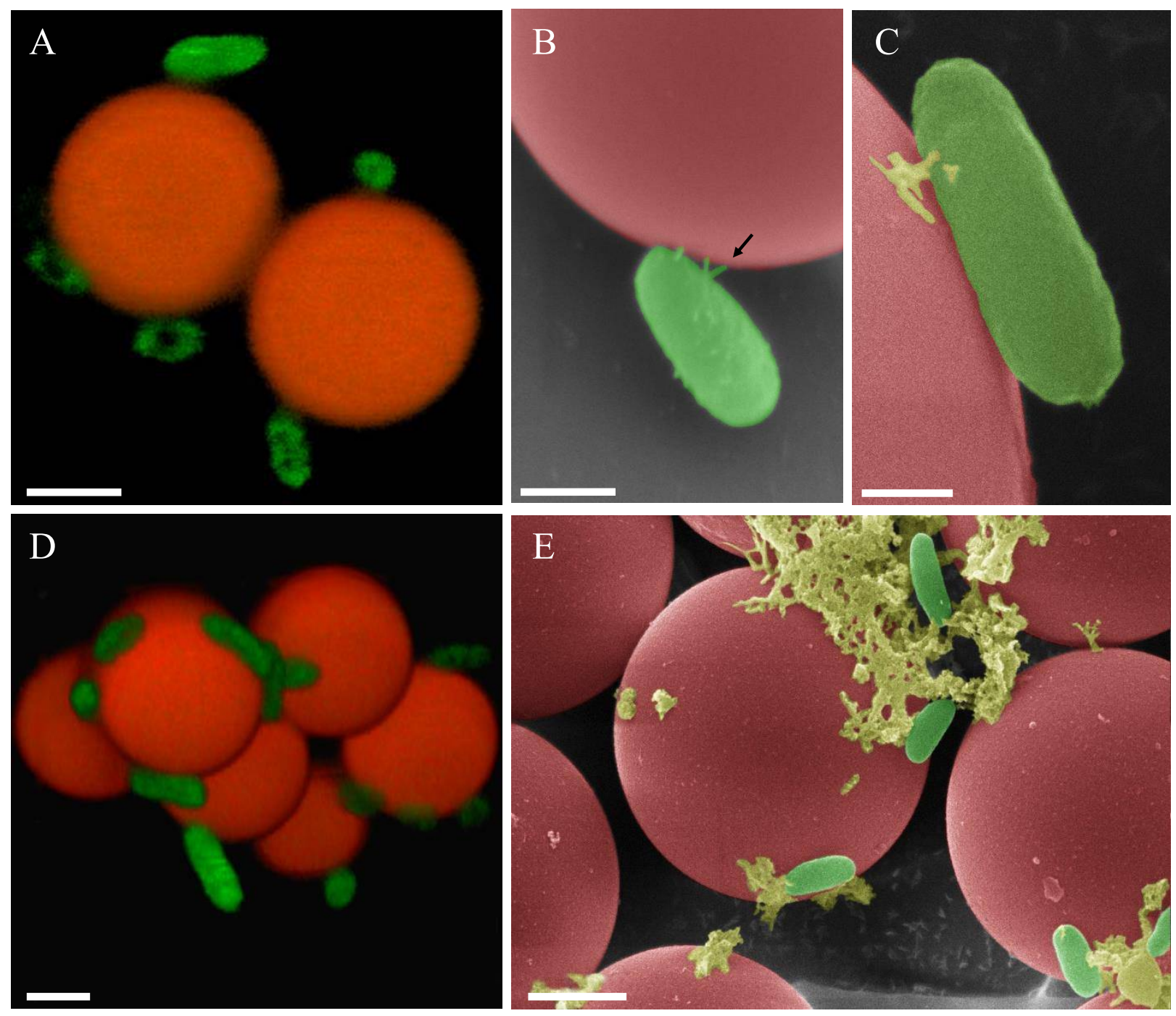


\section{Figure 2}
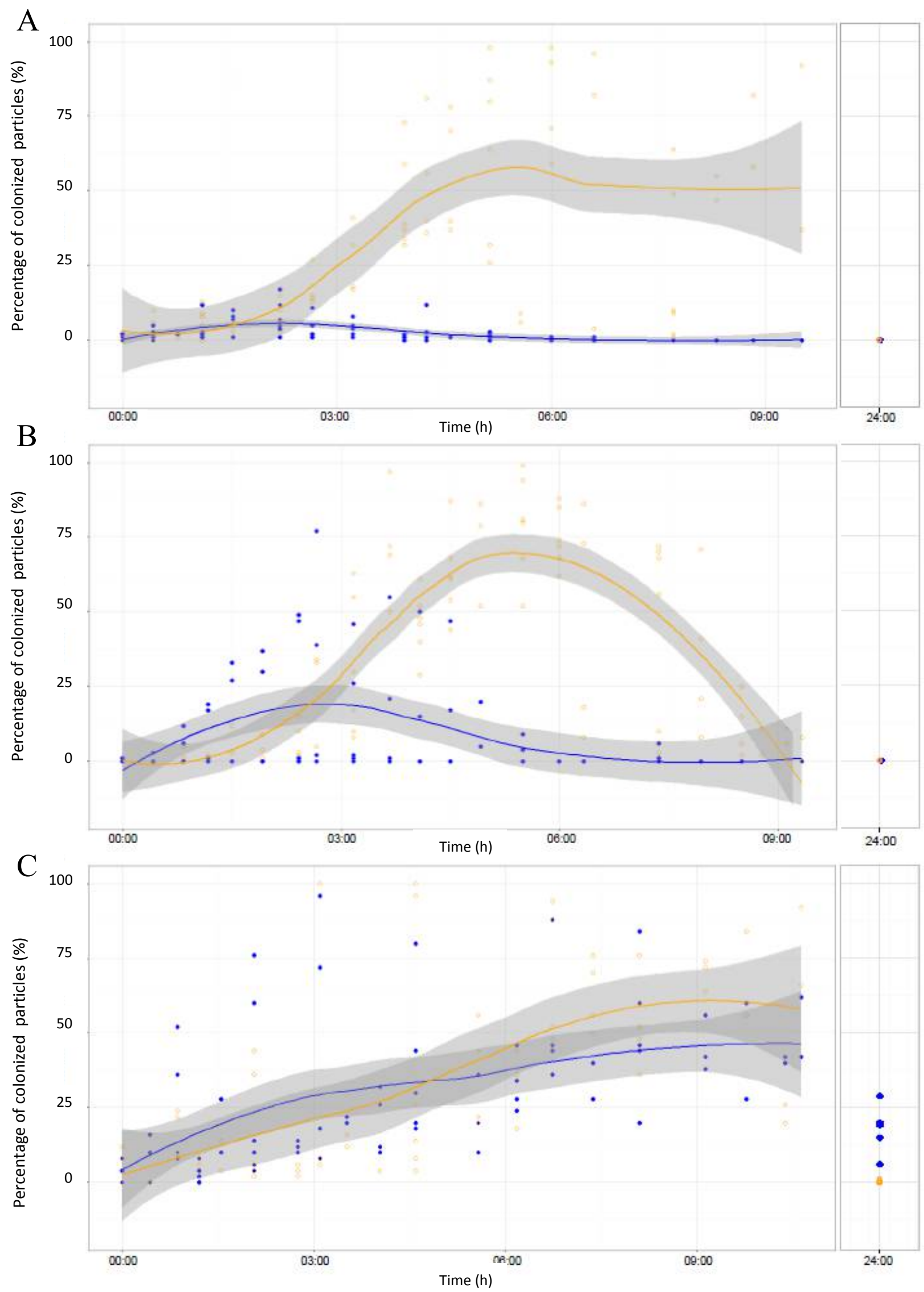


\section{Figure 3}
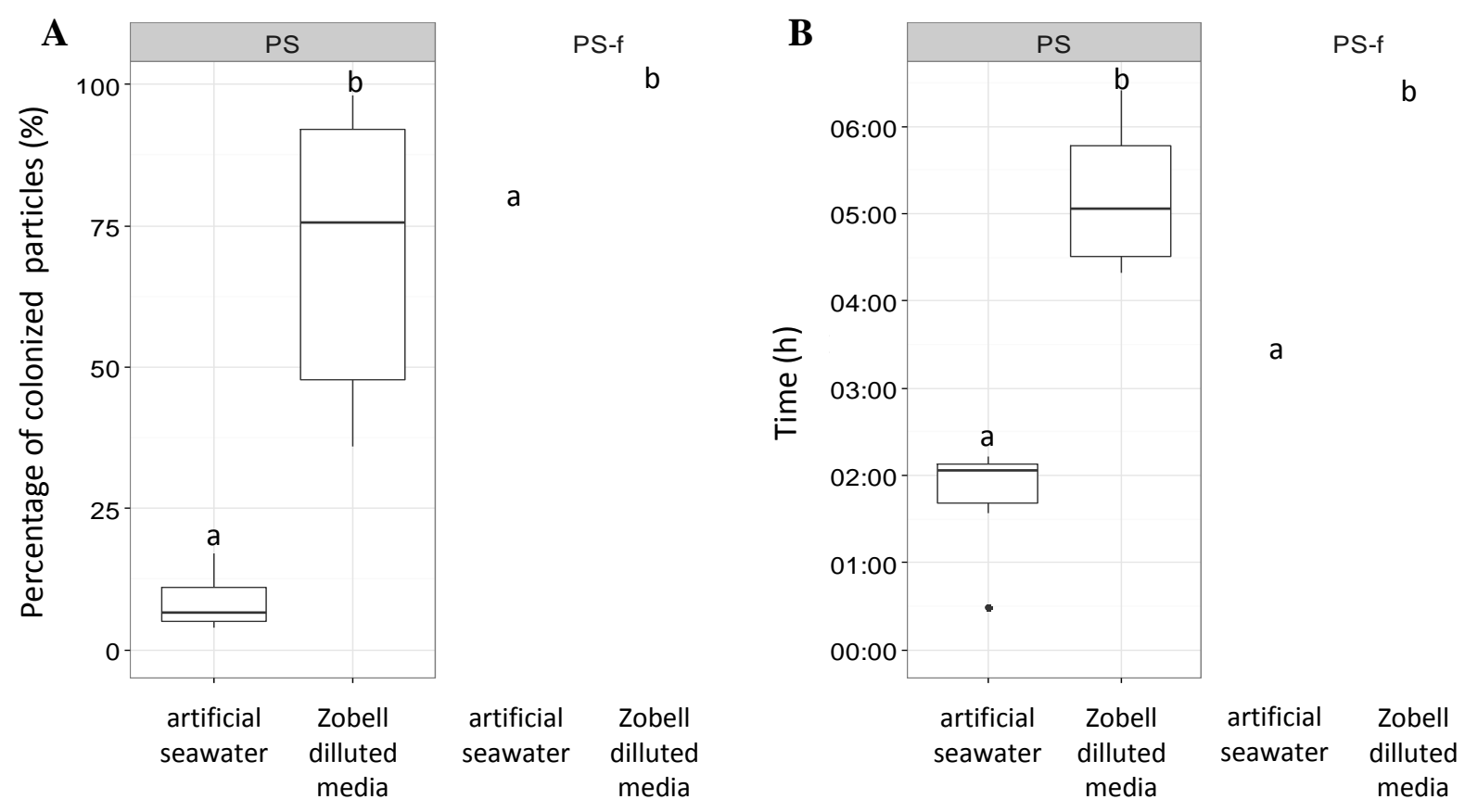


\section{Figure 4}

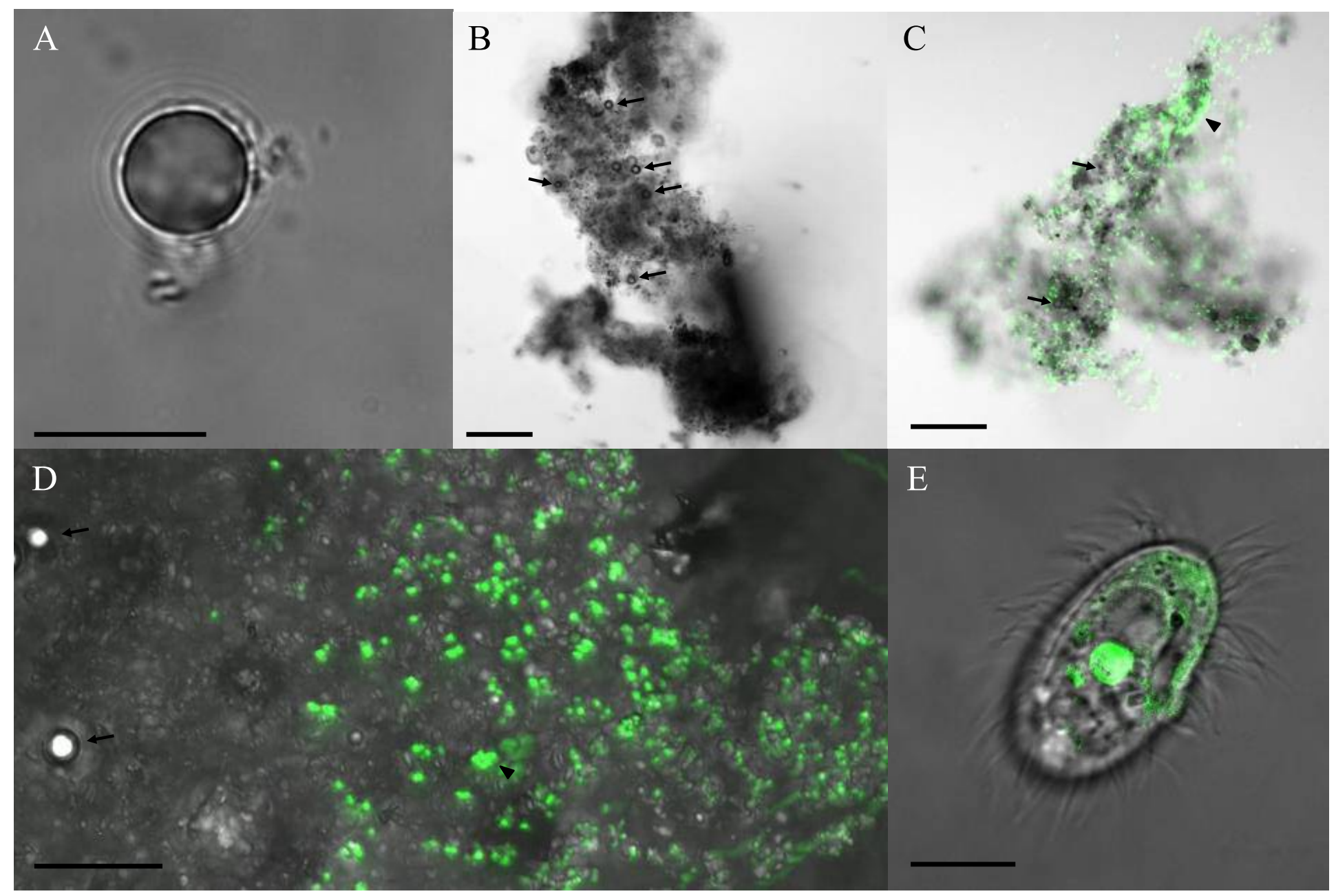

